\title{
Medizinischer Fortschritt: kostspielig oder kostbar?
}

\section{Pierre-François Cuénoud}

Dr. med., Vizepräsident der FMH, Departementsverantwortlicher Tarife und Gesundheitsökonomie Spitalärzte

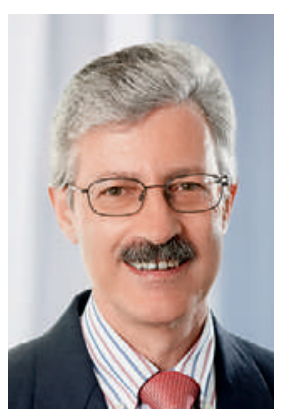

Insbesondere der im stationären Bereich tätigen Ärzteschaft wirft man zunehmend vor, die Gesundheitskosten in die Höhe zu treiben, indem sie den auf ihre Hilfe angewiesenen Patienten immer mehr Leistungen aufdrängen wollen. Lässt sich dies objektiv belegen? Das Gesundheitswesen erlebt eine rasante technologische Entwicklung, die vieles medizinisch möglich macht, wovon man lange nur träumen konnte. Der anfangs meist noch hohe Preis für die anspruchsvolle Technik sinkt dabei nach einigen Jahren deutlich. Die diagnostischen und therapeutischen Vorteile sind in der Regel erheblich. Um ernsthaft Bilanz zu ziehen, ist eine umfassende Kosten-Nutzen-Analyse erforderlich.

Mit strengem Blick auf ihre Kostenansätze haben die Versicherer vor kurzem Kritik an drei unserer Fachgebiete geäussert:

- Unsere Dermatologen führen immer mehr Hautgewebeproben durch. Aber stellt dieses Handeln nicht eine wichtige Massnahme dar, in Anbetracht der Sensibilisierung unserer Bevölkerung für die Gefahren von Melanomen, wo unser Land doch eines der höchsten Vorkommen weltweit aufweist?

- Unsere Augenärzte operieren immer mehr Katarakte. Da unsere Bevölkerung immer älter wird, nimmt dieses Leiden zwangsläufig zu. Der graue Star ist aber kein unabwendbares Schicksal, sondern lohnt eine Behandlung auch bei älteren Menschen, damit diese länger ihre Selbstständigkeit bewahren können und weniger Gefahr laufen zu stürzen.

Es müssen diagnostische und therapeutische Leitlinien festgesetzt werden, die sich an den Grundsätzen von "choosing wisely" orientieren und gleichzeitig eine Kontrolle der Indikationsqualität gewährleisten.

- Unsere Radiologen wenden immer mehr bildgebende Verfahren an. Dank ihrer Qualität und Präzision erlauben diese Verfahren sehr genaue Untersuchungen; häufig können zu rein diagnostischen Zwecken durchgeführte chirurgische Eingriffe dadurch vermieden werden.

Natürlich lässt sich nicht bestreiten, dass manchmal über das Ziel hinausgeschossen wird. Zwar wird in die- sem Zusammenhang das DRG-Vergütungsprinzip im stationären Bereich häufig als unseliger Anreiz genannt - derzeit lässt sich diese Behauptung aber kaum aufrechterhalten (siehe den Artikel von B. Meyer und B. Rohner auf Seite 1082-1083). Andererseits ist der gesetzliche Rahmen vielfältig: Während KVG und UVG sehr streng sind, verleitet das VVG auf dem freien Markt der Zusatzversicherungen zu Übertreibungen.

Das Gesundheitswesen erlebt eine rasante technologische Entwicklung, die vieles medizinisch möglich macht, wovon man lange nur träumen konnte.

Angesichts dieses Dilemmas ist es Aufgabe der Ärzteschaft, vernünftige Spielregeln zu bestimmen und anzuwenden. Es müssen diagnostische und therapeutische Leitlinien festgesetzt werden, die es ermöglichen, eine der Situation des einzelnen Patienten angemessene Entscheidung zu treffen ("choosing wisely»), und die gleichzeitig eine Kontrolle der Indikationsqualität gewährleisten. Neue Techniken müssen in Universitätszentren entwickelt und geprüft werden. Die unkontrollierte Verbreitung und unsachgemässe Nutzung technologischer Schmuckstücke ist dagegen zu vermeiden.

In den letzten zehn Jahren hat das Gesundheitswesen in unserem Land für den grössten Zuwachs an Arbeitsplätzen gesorgt. Das Gesundheitswesen bietet eine Reihe attraktiver Berufe und schafft mit seiner Dynamik Wohlstand. Die erbrachten Leistungen tragen eindeutig zum Erhalt der Lebensqualität unserer Bevölkerung bei. Um die öffentliche Gesundheit zu fördern und dabei einen wirksamen Einsatz der medizinischen Möglichkeiten zu sichern, müssen die politischen Entscheidungsträger innerhalb eines vernünftigen Zeitraums eine adäquate Vergütung der bewährten Leistungen ermöglichen, zugunsten derjenigen Einrichtungen, die diese Leistungen zu angemessenen Bedingungen anbieten. Der medizinische Fortschritt mag mitunter kostspielig sein, er bietet aber einen enormen Nutzen. Dieser Umstand sollte bei der Wahl des ökonomischen Ansatzes gebührend berücksichtigt werden, anstatt den Fortschritt und die damit einhergehenden Investitionen zu behindern. 